\title{
Predictors of "occult" intra-abdominal injuries in blunt trauma patients
}

\section{Indicadores de lesões intra-abdominais "ocultas" em pacientes vítimas de trauma fechado admitidas sem dor abdominal ou alterações no exame físico do abdome}

\author{
José Gustavo Parreira, TCBC-SP; Juliano Mangini Dias Malpaga²; Camilla Bilac Olliarl2; Jacqueline A. G. Perlingeiro, TCBC-SP;
} Silvia C. Soldá, TCBC-SP'; José Cesar Assef, TCBC-SP'1

\author{
A B S T R A C T
}

\begin{abstract}
Objective: to assess predictors of intra-abdominal injuries in blunt trauma patients admitted without abdominal pain or abnormalities on the abdomen physical examination. Methods: We conducted a retrospective analysis of trauma registry data, including adult blunt trauma patients admitted from 2008 to 2010 who sustained no abdominal pain or abnormalities on physical examination of the abdomen at admission and were submitted to computed tomography of the abdomen and/or exploratory laparotomy. Patients were assigned into: Group 1 (with intra-abdominal injuries) or Group 2 (without intra-abdominal injuries). Variables were compared between groups to identify those significantly associated with the presence of intra-abdominal injuries, adopting $p<0.05$ as significant. Subsequently, the variables with $p<0.20$ on bivariate analysis were selected to create a logistic regression model using the forward stepwise method. Results: A total of 268 cases met the inclusion criteria. Patients in Group I were characterized as having significantly $(p<0.05)$ lower mean AIS score for the head segment $(1.0 \pm 1.4$ vs. $1.8 \pm 1.9)$, as well as higher mean AIS thorax score $(1.6 \pm 1.7$ vs. $0.9 \pm 1.5)$ and ISS $(25.7 \pm 14.5$ vs. $17,1 \pm 13,1)$. The rate of abdominal injuries was significantly higher in run-over pedestrians $(37.3 \%)$ and in motorcyclists $(36.0 \%)(p<0.001)$. The resultant logistic regression model provided $73.5 \%$ accuracy for identifying abdominal injuries. The variables included were: motorcyclist accident as trauma mechanism $(p<0.001-$ OR $5.51 ; 95 \% \mathrm{Cl} 2.40-12.64)$, presence of rib fractures $(p<0.003-O R 3.00 ; 95 \% \mathrm{Cl} 1.47-6.14)$, run-over pedestrian as trauma mechanism ( $p=0.008-O R 2.85 ; 95 \% \mathrm{Cl} 1.13-6.22)$ and abnormal neurological physical exam at admission ( $p=0.015-$ OR 0.44; $95 \% \mathrm{Cl}$ 0.22-0.85). Conclusion Intra-abdominal injuries were predominantly associated with trauma mechanism and presence of chest injuries.
\end{abstract}

Key words: Diagnosis. Delayed Diagnosis. External Causes. Multiple Trauma. Abdominal Injuries.

\section{INTRODUCTION}

B lunt trauma is commonly seen in large urban centers, largely due to road traffic accidents, falls and interpersonal violence. The frequency of abdominal injuries varies according to sample analyzed ${ }^{1,2}$. In studies including minor trauma the rate of these injuries is typically less than $10 \%^{1,2}$. A number of different factors can add to the difficulty in diagnosing intra-abdominal injuries, including changes in level of consciousness, distracting injuries, sedation and use of analgesics ${ }^{1-4}$.

These injuries are not initially diagnosed in a substantial proportion of patients. Delayed diagnosis can have serious consequences and may even lead to "preventable" deaths ${ }^{5-8}$. Therefore, several diagnostic modalities have evolved in the past three decades: diagnostic peritoneal lavage, ultrasound, computed tomography (CT) and videolaparoscopy, each having inherent advantages, disadvantages and complications ${ }^{9}$.

The most accurate imaging exam is computed tomography, being able to identify most injuries ${ }^{10}$. Some authors advocate liberal use of CT in blunt trauma patients ${ }^{11}$. However, the exam poses some risks to the patient such as anaphylactic reactions due to administration of contrast and cancers resulting from radiation exposure ${ }^{12,13}$. Missed abdominal injuries can occur despite adherence to strict evaluation protocols and are often associated with the absence of abdominal pain or of abnormalities on the abdomen physical examination ${ }^{4}$.

We believe it is possible to identify variables that characterize patients in whom the presence of intra-abdominal injuries is statistically more likely. The literature refers to these variables as predictors of abdominal injuries ${ }^{14}$. Previous studies have suggested a range of predictors, such

1. Departamento de Cirurgia da Faculdade de Ciências Médicas da Santa Casa de São Paulo, Brasil; 2. Faculdade de Ciências Médicas da Santa Casa de São Paulo, Brasil. 
as the presence of arterial hypotension, metabolic acidosis, severe injuries to the thoracic segment, as well as fractures to the pelvis, long bones and lumbar spine ${ }^{1,14-16}$. However, we found no specific studies assessing these predictors in trauma patients admitted without abdominal pain or changes on physical examination of the abdomen - in whom the risk of diagnostic failure is high. The objective of this study was to identify predictors of abdominal injuries in this specific group of trauma patients.

\section{METHODS}

This study was submitted to the Ethics in Research Committee of the institution and approved under number 443.723.

We conducted a retrospective study of information from medical charts and trauma registry, including blunt trauma patients over the age of 13 years admitted to the Emergency Room of the Irmandade da Santa Casa de Misericórdia de São Paulo between 2008 and 2010. Prospective data collection was performed over this period for all trauma patients over the age of 13 admitted to the emergency room. The data collected was held in a Microsoft Access database.

We assessed data on identification, trauma mechanism, pre-hospital care, vital signs at admission, trauma scores, complementary exams ordered, associated diseases, injuries diagnosed and treatment. This information was routinely collected for all trauma patients admitted to the trauma room, and recorded on standard forms and patient medical charts.

The abdominal assessment protocol for imaging exams routinely employed at the our service entails selective use of Focused Assessment with Sonography for Trauma (FAST), complete abdominal ultrasound (US) and computed tomography $(\mathrm{CT})$ according to the abdominal injury risk assessment by the attending physician. In addition to imaging exams, laboratory assays such as leukocyte count, amylase level and arterial blood gases were performed to screen for possible abdominal injuries. Leukocytosis, elevated amylase and metabolic acidosis are suggestive of injuries that may have been missed by imaging exams.

This study included all blunt trauma patients aged over 13 who underwent computed tomography of the abdomen and/or exploratory laparotomy and that had no abdominal pain or abnormalities on physical examination of the abdomen at admission.

Severity of the sample was stratified using the following trauma measures: Glasgow Coma Scale (GCS) ${ }^{17}$, Revised Trauma Score (RTS) ${ }^{18}$, Abbreviated Injury Scale $(A I S)^{19}$, Organ Injury Scale ${ }^{20}$, and the Injury Severity Score $(\text { ISS })^{21}$. Injuries with an AIS score $\geq 3$ were considered severe. Patients presenting with free intraperitoneal fluid, retroperitoneal hematomas (with or without spinal fractures) and/or injuries to the abdominal wall, but without injuries to specific intra-abdominal anatomic structures, were defined as without intra-abdominal injury (IAI).

Patients were categorized into two groups: Group 1: with intra-abdominal injuries; Group 2: without intraabdominal injuries. Variables for the two groups were compared to identify those significantly associated with the presence of intra-abdominal injuries, using the software Statistical Package for Social Sciences $®$. We first carried out a Bivariate analysis comparing the variables between groups. The Chi-square and Fisher's tests were employed for nominal variables, whereas the Student's t-test was used for quantitative variables. A value of $p<0.05$ was considered significant. Subsequently, the most important variables for diagnosing intra-abdominal injuries in the emergency room (except those depending on performance of abdominal ultrasound) with $p<0.20$ on bivariate analysis were selected to create a logistic regression model using the forward stepwise method.

\section{RESULTS}

During the study period, a total of 5,785 blunt trauma patients were attended, of which 5,202 (89.9\%) presented no abdominal pain or abnormalities on physical examination of the abdomen at admission. Of these, $268(5.1 \%)$ were submitted to computed tomography scan and/or exploratory laparotomy. In this group, age ranged from 14 to 98 years (mean $38.1 \pm 16.1$ ) and $219(81.7 \%)$ were male. Mean systolic arterial pressure, Glasgow coma scale, respiration rate and heart rate at admission were, respectively, $119.7 \pm 36.4 \mathrm{mmHg}$, $11.4 \pm 4.4,17.0 \pm 9.0 \mathrm{ipm}$ and $92.2 \pm 21.6 \mathrm{bpm}$. Mean RTS and ISS calculated for the sample were $6.64 \pm 1.8$ and $19.5 \pm 14.0$, respectively.

The most common trauma mechanisms were runover pedestrians in 90 (33.6\%) cases, accidents involving motorcyclists in 61 (22.8\%), falls from height in 56 (20.9\%), automobile accidents involving occupants of four-wheeled vehicles in 31 (11.6\%), assault in $16(6.0 \%)$ and fall from the standing height in 7 (2.6\%). The remaining 7 (2.6\%) cases had either multiple trauma mechanisms or could not be categorized into any of the above-mentioned groups.

Injuries to the head segment were identified in $131(48.8 \%)$ cases, thorax in 91 (34.0\%), abdomen in 75 $(28.0 \%)$ and extremities in $133(53.4 \%)$. Thirty-seven patients $(13.8 \%)$ had pelvic fractures. Severe injuries (AIS $\geq 3$ ) were detected in the head segment, thorax, abdomen, and extremities in 96 (35.8\%), 75 (28.0\%), 50 (18.7\%) and 107 (39.9\%), respectively.

FAST was performed in 69 of the 75 patients with intra-abdominal injuries, proving positive in 21 cases (30.4\%). Complete abdominal ultrasound was performed in 38 of the 75 patients with intra-abdominal injuries, disclosing positive results in $34(89.5 \%)$ cases. Abdominal computed tomography scans were performed in 66 of the 
75 patients with intra-abdominal injuries, disclosing positive results in $64(89.5 \%)$ cases.

The most frequent abdominal injuries were: splenic in $34(12.7 \%)$ patients; hepatic in $33(12.3 \%)$; and renal in 9 (3.3\%). Injuries to the small intestine and colon were seen in 4 patients, representing $1.4 \%$ of the overall sample (Table 1). A total of $31(11.6 \%)$ exploratory laparotomies were carried out, 15 (48.0\%) of which were non-therapeutic. Abdominal surgical procedures performed were: splenectomy (8), diaphragm suture (3), enterorrhaphy (3), bladder suture (1), renal suture (1), enterectomy/ anastomosis (1), ligation of the common iliac vein (1) and revascularization of the common iliac artery (1).

Comparison of the numerical variables between groups revealed that patients with abdominal injuries (Group I) exhibited significantly lower mean AIS score for the head segment (1.0 \pm 1.4 vs. $1.8 \pm 1.9$ ) and higher mean AIS thorax score $(1.6 \pm 1.7$ vs. $0.9 \pm 1.5)$ and ISS $(25.7 \pm 14.5$ vs. 17.1 \pm 13.1 ) than Group II (Table 2). We observed no significant difference between the groups as for mean systolic arterial pressure at admission, respiratory rate at admission, Glasgow coma scale score at admission, heart rate at admission, RTS, age or AIS in extremities.

A significant difference in trauma mechanism between the groups was detected $(p<0.001)$. The rate of abdominal injuries was significantly greater in individuals run over by road vehicles (37.3\%) and among motorcyclists $(36.0 \%)$, compared to those suffering falls from height $(13.3 \%)$, automobile accidents (9.3\%), assault (1.3\%) and falls from standing height (1.3\%).

A significant difference was found $(p<0.05)$ between groups as for frequency of chest drainage at admission ( $25.3 \%$ vs. $10.4 \%)$, abnormal pelvic radiograph $(24.0 \%$ vs. $11.4 \%)$, brain swelling $(0.7 \%$ vs. $6.7 \%)$, traumatic subarachnoid hemorrhage ( $6.7 \%$ vs. $12.4 \%)$, skull fracture $(0.7 \%$ vs. $7.1 \%)$, hemothorax $(24.0 \%$ vs. $14.0 \%)$, pneumothorax (25.3\% vs. $9.3 \%$ ), rib fractures (37.3\% vs. $17.6 \%)$, flail chest (20.0\% vs. $6.3 \%)$, lung contusion $(28.0 \%$ vs. $11.4 \%)$, chest drainage (33.3\% vs. $15.0 \%)$, and pelvic fractures $(26.7 \%$ vs. $8.8 \%)$ (Table 3$)$. Thirtythree $(12.3 \%)$ patients died, but there was no difference in lethality between groups (10.7\% vs. 13.0\%).

Table 1 - Intra-abdominal injuries detected in 75 blunt trauma patients admitted without abdominal pain or changes on physical examination of the abdomen (Group 1), according to AAST-OIS grade.

\begin{tabular}{|c|c|c|c|c|c|c|c|}
\hline & 1 & II & III & IV & V & & Total \\
\hline Spleen & 8 & 10 & 8 & 5 & 3 & 34 & $(45.3 \%)$ \\
\hline Liver & 7 & 13 & 9 & 3 & 1 & 33 & $(44.0 \%)$ \\
\hline Kidneys & 1 & 3 & 3 & 0 & 2 & 9 & $(12.0 \%)$ \\
\hline Small intestine/colon & 0 & 2 & 1 & 1 & 0 & 4 & $(5.3 \%)$ \\
\hline Diaphragm & 0 & 0 & 3 & 0 & 0 & 3 & $(4.0 \%)$ \\
\hline Bladder & 0 & 1 & 1 & 0 & 0 & 2 & $(2.6 \%)$ \\
\hline Abdominal vessels & 0 & 0 & 0 & 1 & 0 & 1 & $(1.3 \%)$ \\
\hline
\end{tabular}

Source: records and trauma registry of the Pronto Socorro Central da Irmandade da Santa Casa de Misericórdia de São Paulo (2008/2010).

Table 2 - Comparison of numerical variables between groups.

\begin{tabular}{|c|c|c|c|c|c|}
\hline Variables & $p$ & Mean & $\begin{array}{l}\text { Sroup I } \\
\mathrm{N}=75 \\
\text { Standard Deviation }\end{array}$ & Mean & $\begin{array}{l}\text { Group II } \\
\mathrm{N}=193 \\
\text { Standard Deviation }\end{array}$ \\
\hline Age (years) & 0.593 & 37.2 & 38.4 & 38.4 & 16.3 \\
\hline SAP at admission $(\mathrm{mmHg})$ & 0.111 & 113.9 & 122.1 & 122.1 & 35.5 \\
\hline Respiratory rate & 0.139 & 18.4 & 9.7 & 16.4 & 8.6 \\
\hline Heart rate (bpm) & 0.566 & 93.5 & 91.7 & 91.7 & 22.9 \\
\hline Glasgow Coma Scale & 0.120 & 12.3 & 10.9 & 10.9 & 4.5 \\
\hline AIS in head & $<0.001$ & 1.0 & 1.4 & 1.8 & 1.9 \\
\hline AIS in thorax & 0.001 & 1.6 & 1.7 & 0.9 & 1.5 \\
\hline AIS in extremities & 0.869 & 1.7 & 1.6 & 1.6 & 1.8 \\
\hline ISS & $<0.001$ & 25.7 & 14.5 & 17.1 & 13.1 \\
\hline RTS & 0.730 & 6.7 & 6.6 & 6.6 & 1.8 \\
\hline TRISS & 0.598 & 0.97 & 0.9 & 0.95 & 0.1 \\
\hline
\end{tabular}

Source: records and trauma registry of the Pronto Socorro Central da Irmandade da Santa Casa de Misericórdia de São Paulo (2008/2010). SAP: Systolic arterial pressure; AIS: Abbreviated Injury Scale; ISS: Injury Severity Score; RTS: Revised Trauma Score; TRISS: Trauma And Injury Severity Score. 
Similarly, no significant difference was found between the groups in terms of frequency of orotracheal intubation at admission, extradural hematoma, subdural hematoma, brain contusion, diffuse axonal injury, skull base fracture, spinal cord trauma, upper and lower limb fractures, or open fractures of the upper and lower limbs (Table 3).

The following variables were input to the logistic regression model: systolic arterial pressure at admission, respiratory rate at admission, Glasgow coma scale score at admission, abnormal neurological physical exam, abnormal physical exam of the thoracic region, rib fractures, abnormal pelvic radiograph, abnormal chest radiograph and trauma mechanisms (run-over pedestrian, motorcyclist, fall from height, fall from standing height, occupant of four-wheeled automobile and assault). The model built using logistic regression yielded $73.5 \%$ accuracy for identifying abdominal injuries. The variables included were: motorcyclist as trauma mechanism $(p<0.001-$ OR $5.51 ; 95 \% \mathrm{Cl} 2.40$ -
12.64), presence of rib fractures $(p<0.003-O R 3.00 ; 95 \% \mathrm{Cl}$ 1.47-6.14), run-over pedestrian as trauma mechanism (0.008 - OR 2.85; 95\% Cl 1.13-6.22) and abnormal neurological physical exam at admission $(p=0.015-$ OR 0.44; 95\%Cl 0.22-0.85).

\section{DISCUSSION}

The devising of a definitive protocol for diagnosing intra-abdominal injuries in blunt trauma patients remains a challenge, since potentially lethal injuries often go undetected on physical, laboratory and imaging exams ${ }^{4}$. Use of the most accurate exam, computed tomography, is becoming increasingly limited, mainly owing to its association with the genesis of malignant tumors ${ }^{12,13}$.

The results of this study in a sample of blunt trauma patients without abdominal pain or altered physical

Table 3 - Comparison of categorical variables between groups.

\begin{tabular}{lccc}
\hline Variable & $\begin{array}{c}\text { Group I (\%) } \\
\text { N=75 }\end{array}$ & $\begin{array}{c}\text { Group II (\%) } \\
\text { N=193 }\end{array}$ & p \\
\hline Orotracheal intubation & 21.3 & 31.6 & 0.095 \\
Chest drainage at admission & 25.3 & 10.4 & 0.007 \\
Abnormal neurological physical exam & 41.3 & 56.5 & 0.025 \\
Abnormal thorax physical exam & 38.7 & 21.2 & 0.004 \\
Abnormal chest radiograph & 54.7 & 28.0 & $<0.001$ \\
Abnormal pelvic radiograph & 24.0 & 11.4 & 0.033 \\
Extradural hematoma & 5.3 & 11.4 & 0.363 \\
Subdural hematoma & 4.0 & 11.4 & 0.097 \\
Traumatic subarachnoid hemorrhage & 6.7 & 12.4 & 0.044 \\
Brain contusion & 5.3 & 7.8 & 0.124 \\
Diffuse axonal injury & 0.7 & 6.7 & 0.343 \\
Brain Swelling & 0.7 & 7.1 & 0.046 \\
Skull fracture & 0.7 & 4.9 & 0.036 \\
Skull base fracture & 2.7 & 6.4 & 0.157 \\
Spinal cord trauma & 5.3 & 8.3 & 0.294 \\
Hemothorax & 24.0 & 14.0 & 0.035 \\
Pneumothorax & 25.3 & 9.3 & 0.003 \\
Rib fracture & 37.3 & 17.6 & 0.001 \\
Flail chest & 20.0 & 6.3 & 0.001 \\
Lung contusion & 28.0 & 11.4 & 0.003 \\
Chest drainage & 33.3 & 15.0 & 0.003 \\
Pelvic fracture & 26.7 & 8.8 & $<0.001$ \\
Upper limb fracture & 17.3 & 14.0 & 0.304 \\
Lower limb fracture & 17.3 & 13.5 & 0.702 \\
Open upper limb fracture & 4.0 & 4.1 & 0.630 \\
Open lower limb fracture & 5.3 & 7.8 & 0.343 \\
Severe injury in head segment & 22.7 & 40.9 & 0.005 \\
Severe injury in thorax segment & 45.3 & 21.2 & 39.9 \\
Severe injuries in extremities & 40.0 & 0.988 \\
Source: records and trauma & 0.3 & \\
\hline
\end{tabular}

Source: records and trauma registry of the Pronto Socorro Central da Irmandade da Santa Casa de Misericórdia de São Paulo (2008/2010). 
exam of the abdomen showed that several clinical variables were significantly associated with the presence of intraabdominal injuries, such as trauma mechanism and presence of thoracic injuries.

It is important to emphasize that the sample investigated in this study represented only a fraction (5.1\%) of the total blunt trauma patients seen at that service during the study period. The trauma scores RTS $(6.64 \pm 1.8)$ and ISS (19.5 \pm 14.0$)$ revealed a more severe group, selected for CT or exploratory laparotomy. The rate of intra-abdominal injuries (28.0\%) was also higher than that observed in other series ${ }^{1,2,9}$. However, in clinical practice most diagnostic doubts about intra-abdominal injuries arise in the situations represented by the sample.

One of the most widely recognized factors hampering diagnosis in blunt trauma patients is impaired consciousness secondary to head trauma or use of sedatives, particularly among subjects submitted to orotracheal intubation at admission ${ }^{22}$. In our study, those patients presenting with abdominal injuries had greater mean GCS score at admission and lower mean AIS score in the head segment. Multivariate analysis of the present sample showed that the presence of abnormal neurological physical exam at admission was inversely correlated with presence of intra-abdominal injuries (OR 0.44; 95\% Cl 0.22-0.85).

However, this finding does not preclude the need for objective abdominal evaluation in severe trauma patients with reduced level of consciousness. It is noteworthy that a previous study involving a sample of unselected blunt trauma patients found greater head trauma severity in patients with intra-abdominal injuries ${ }^{22}$. Comparison against this data reveals that the present study sample contained patients that were more severe with a higher rate of intra-abdominal injuries, possibly explaining the logistic regression results.

Mean thorax AIS, as well as frequencies of hemothorax, pneumothorax, rib fractures and flail chest, were higher in patients with intra-abdominal injuries. This finding was described in previous reports directly associating severity of thoracic trauma with the presence of intra-abdominal injuries $14,15,16$. This marker also held true for the present group of trauma patients, admitted without abdominal pain or altered abdomen physical examination. On the multivariate analysis, the presence of rib fractures was significantly associated with intra-abdominal injuries (OR 3.00; 95\% Cl 1.47-6.14).

It is believed that associated injuries in other body segments, particularly severe injuries, can "mask" the presence of intra-abdominal injuries ${ }^{3}$. In this group of "asymptomatic" patients, both AIS in extremities and the rate of upper and lower limb fractures were not statistically associated with higher frequency of intra-abdominal injuries. The selection of patients apparently influences this finding. In the 2004 study of Gonzales et al. ${ }^{2}$, intra-abdominal injuries were found in only $1.2 \%$ of blunt trauma patients with extra-abdominal injuries requiring surgery. By contrast, a high percentage of cases had severe intra-ab- dominal injuries requiring surgical treatment in our sample. This highlights the need for objective investigation of the abdomen even in asymptomatic cases.

Notably, the rate of pelvic fractures was higher in patients with intra-abdominal injuries, corroborating the findings of other studies ${ }^{15,16,22}$. Pelvic fractures appear to be a marker of severity in trauma, and intra-abdominal injuries should always be actively investigated in these cases. On multivariate analysis however, we detected no significant association between abnormal radiographs of the pelvis and abdominal injuries, most likely owing to sample selection.

We found no difference between groups for vital signs data at admission. In other studies, arterial hypotension was identified as a marker of the presence of intra-abdominal injuries in blunt trauma patients ${ }^{14,16,22}$. When analyzing unselected blunt trauma patients, Glasgow Coma Scale score, heart and respiratory rates and RTS have been shown to be significantly associated with the presence of intraabdominal injuries ${ }^{22}$. This is perhaps the most important finding in the present study. In the cases with absence of abdominal pain and normal abdominal physical exam, vital signs data did not serve as a discriminating parameter for the detection of intra-abdominal injuries in this selected sample of severe patients with a high frequency of associated injuries.

Trauma mechanism was also a variable included in the multivariate analysis using logistic regression. Both motorcyclists involved in accidents (OR 5.51; 95\% Cl 2.4012.64) and run-over pedestrians (OR 2.85; 95\% Cl 1.13-6.22) had a higher likelihood of sustaining intra-abdominal injuries. In an earlier study involving unselected blunt trauma patients, a significant association was found between motorcyclists and severe intra-abdominal injuries ${ }^{23}$.

Some authors have proposed a combination of variables to determine risk scores for intra-abdominal injuries $^{1,15,24,25}$, indicating the use of complementary exams. Specific scores were proposed for children and adults, with optimized areas under the ROC curve. Studies validating these scores in a homogeneous population are currently lacking. We found no similar proposals for blunt trauma patients without abdominal pain or altered abdomen exam.

A retrospective analysis such as this has some limitations. In order to ensure a "true negative" as a basis for comparison, we chose to include only individuals submitted to exploratory laparotomy and/or computed tomography of the abdomen and pelvis. This would decrease the "false negative" rate. However, both procedures depend on subjective analysis. Despite the common understanding that patients sustaining diffuse peritonitis, uncontrolled abdominal hemorrhage, diaphragmatic hernias or intraperitoneal bladder rupture should be best treated with surgical exploration, other situations could raise discussion about the best option. The same is observed in the $\mathrm{CT}$ protocols for abdominal assessment. There are several options and many depend 
on personal interpretation. From a population of 5,202 blunt trauma patients, only $5 \%$ were included and, certainly, this sample does not represent the entire population of blunt trauma victims.

A consequence of this selection was the possibility of non-inclusion in the sample of some patients whose intraabdominal injury was not diagnosed for not having undergone CT or exploratory laparotomy. There were no reports, however, of readmissions to the hospital for complications of undetected intra-abdominal injuries during the study period. Nonetheless, this does not rule out the possibility of minor injuries which did not manifest clinically.

The standardization of the physical exam of the abdomen may also draw criticism. However, not only resident doctors admitted the patients but also attending physicians from the service. The inclusion of trauma patients with impaired consciousness and/or arterial hypotension in this study may also raise questions, since these are high- risk patients for suspected intra-abdominal injuries despite a normal abdominal physical exam. Nevertheless, even in this specific group of patients there is a risk of undiagnosed injuries, with subsequent serious consequences. Inclusion of these cases was therefore deemed important.

A final analysis of the data clearly evidences the association of trauma mechanisms and chest injuries with the presence of intra-abdominal injuries in this group of blunt trauma patients admitted without abdominal pain or abnormalities on physical examination of the abdomen. Other classic predictors of intra-abdominal injuries described in unselected blunt trauma patients, such as systolic arterial pressure at admission and presence of severe injuries to the extremities, among others, were not associated with intra-abdominal injuries in the sample studied. This absence of association might be explained by the anatomical and physiological severity of the patients selected for this study.

\title{
R E S U M O
}

\begin{abstract}
Objetivo: avaliar os indicadores de lesões intra-abdominais em vítimas de trauma fechado admitidas sem dor abdominal ou alterações no exame físico do abdome. Método: estudo retrospectivo das vítimas de trauma fechado com idade superior a 13 anos, admitidas no período de 2008-2010. Selecionamos para estudo todos que foram submetidos à tomografia computadorizada de abdome elou laparotomia exploradora e que, à admissão, não apresentavam dor abdominal ou alterações ao exame físico do abdome. Os doentes foram separados em: Grupo 1 (com lesões intra-abdominais) e Grupo 2 (sem lesões intra-abdominais). As variáveis foram comparadas entre os grupos, considerando $p<0,05$ como significativo. Em um segundo passo, selecionamos as variáveis com $p<0,20$ na análise bivariada para criar modelo de regressão logística pelo método forward stepwise. Resultados: foram incluídos 268 casos. Os doentes com lesão abdominal caracterizaram-se por apresentar, significativamente $(p<0,05)$, menor média de AIS em segmento cefálico (1,0 $\pm 1,4$ vs. 1,8 $\pm 1,9)$, bem como, maior média de AIS em tórax $(1,6 \pm 1,7$ vs. 0,9 $\pm 1,5)$ e de ISS $(25,7 \pm 14,5$ vs. $17,1 \pm 13,1)$. A frequência de lesões abdominais foi significativamente maior nas vítimas de atropelamentos $(37,3 \%)$ e motociclistas (36\%) $(p<0,001)$. A regressão logística construiu um modelo utilizando as seguintes variáveis: motociclista como mecanismo de trauma ( $p<0,001-O R=5,51 ; I C 95 \% 2,40-12,64)$, presença de fraturas de costelas $(p<0,003-0 R=3,00 ; I C 95 \%$ 1,47-6,14), atropelamento como mecanismo de trauma ( $p=0,008-O R=2,85 ;$ IC95\% 1,13-6,22) e exame físico neurológico anormal a admissão ( $p=0,015$ - OR=0,44; IC95\% 0,22-0,85). Conclusão: as lesões intra-abdominais foram relacionadas principalmente com o mecanismo de trauma e a presença de lesões torácicas.
\end{abstract}

Descritores: Diagnóstico. Diagnóstico Tardio. Causas Externas. Traumatismo Múltiplo. Traumatismos Abdominais.

\section{REFERENCES}

1. Poletti PA, Mirvis SE, Shanmuganathan K, Takada T, Killeen KL, Perlmutter $D$, et al. Blunt abdominal trauma patients: can organ injury be excluded without performing computed tomography? J Trauma. 2004;57(5):1072-81.

2. Gonzalez RP, Han M, Turk B, Luterman A. Screening for abdominal injury prior to emergent extra-abdominal trauma surgery: a prospective study. J Trauma. 2004;57(4):739-41.

3. Ferrera PC, Verdile VP, Bartfield JM, Snyder HS, Salluzzo RF. Injuries distracting from intraabdominal injuries after blunt trauma. Am J Emerg Med. 1998;16(2):145-9.

4. Jones EL, Stovall RT, Jones TS, Bensard DD, Burlew CC, Johnson JL, et al. Intra-abdominal injury following blunt trauma becomes clinically apparent within 9 hours. J Trauma Acute Care Surg. 2014;76(4):1020-3.

5. Sung $\mathrm{CK}, \mathrm{Kim} \mathrm{KH}$. Missed Injuries in abdominal trauma. J Trauma. 1996;41(2):276-82.
6. Crookes BA, Shackford SR, Gratton J, Khaleel M, Ratliff J, Osler T. "Never be wrong": the morbidity of negative and delayed laparotomies after blunt trauma. J Trauma. 2010;69(6):1386-91; discussion 1391-2.

7. Eren B, Türkmen N, Gündo mu ÜN. Delayed spleen rupture after blunt abdominal trauma (case report). Georgian Med News. 2012;(206):22-4

8. Ertugrul G, Coskun M, Sevinc M, Ertugrul F, Toydemir T. Delayed presentation of a sigmoid colon injury following blunt abdominal trauma: a case report. J Med Case Rep. 2012;6:247.

9. Nishijima DK, Simel DL, Wisner DH, Holmes JF. Does this adult patient have a blunt intra-abdominal injury? JAMA. 2012;307(14):1517-27.

10. Sise MJ, Kahl JE, Calvo RY, Sise CB, Morgan JA, Shackford $S R$, et al. Back to the future: reducing reliance on torso computed tomography in the initial evaluation of blunt trauma. J Trauma Acute Care Surg. 2013;74(1):92-7; discussion 97-9. 
11. Deunk J, Brink M, Dekker HM, Kool DR, van Kuijk C, Blickman JG, et al. Routine versus selective computed tomography of the abdomen, pelvis, and lumbar spine in blunt trauma: a prospective evaluation. J Trauma. 2009;66(4):1108-17.

12. Zallman L, Woolhandler S, Himmelstein D, Bor DH, McCormick D. Computed tomography associated cancers and cancer deaths following visits to U.S. emergency departments. Int J Health Serv. 2012;42(4):591-605

13. Printz C. Computed tomography scans and cancer risk: the latest findings. Cancer. 2013;119(4):701-2

14. Mackersie RC, Tiwary AD, Shackford SR, Hoyt DB. Intra-abdominal injury following blunt trauma. Identifying the high-risk patient using objective risk factors. Arch Surg. 1989;124(7):809-13.

15. Deunk J, Brink M, Dekker HM, Kool DR, Blickman JG, van Vugt $A B$, et al. Predictors for the selection of patients for abdominal $C T$ after blunt trauma: a proposal for a diagnostic algorithm. Ann Surg. 2010;251(3):512-20.

16. Beck D, Marley R, Salvator A, Muakkassa F. Prospective study of the clinical predictors of a positive abdominal computed tomography in blunt trauma patients. J Trauma. 2004;57(2):296300.

17. Teasdale G, Jennet B. Assessment of coma and impaired consciousness: a pratical scale. Lancet. 1974;2(7872):81-4.

18. Champion HR, Sacco WJ, Copes WS, Gann DS, Gennarelli TA, Flanagan ME. A revision of the Trauma Score. J Trauma. 1989;29(5):623-9.

19. Association for the Advancement of Automotive Medicine (USA), Committee on Injury Scaling. The abbreviated injury scale-1990 Revision (AIS-90). Des Plaines, IL: Association for the Advancement of Automotive Medicine, 1990.
20. Moore EE, Moore FA. American Association for the Surgery of Trauma Organ Injury Scaling: 50th anniversary review article of the Journal of Trauma. J Trauma. 2010;69(6):1600-1.

21. Baker SP, O'Neill B, Haddon W Jr, Long WB. The injury severity score: a method for describing patients with multiple injuries and evaluating emergency care. J Trauma. 1974;14(3):187-96.

22. Farrath S, Parreira JG, Perlingeiro JAG, Soldá SC, Assef JC. Fatores preditores de lesões abdominais em vítimas de trauma fechado. Rev Col Bras Cir. 2012:39(4):295-301.

23. Farrath $S$, Parreira JG, Olliari CB, Silva MA, Perlingeiro JAG, Soldá SC, et al. Identificação de lesões abdominais graves na avaliação inicial das vítimas de trauma fechado. Rev Col Bras Cir. 2013:40(4):305-11

24. Holmes JF, Wisner DH, McGahan JP, Mower WR, Kuppermann N. Clinical prediction rules for identifying adults at very low risk for intra-abdominal injuries after blunt trauma. Ann Emerg Med. 2009;54(4):575-84

25. Shojaee M, Faridaalaee G, Yousefifard M, Yaseri M, Arhami Dolatabadi A, Sabzghabaei A, et al. New scoring system for intraabdominal injury diagnosis after blunt trauma. Chin J Traumatol. 2014;17(1):19-24.

Received at: 15/12/2014

Accepted for publication: 13/02/2015

Conflict of interest: none.

Source of funding: none.

\section{Mailing address:}

José Gustavo Parreira.

E-mail: jgparreira@uol.com.br. 